\title{
Impact of transmitter phase noise on NFDM transmission with discrete spectral modulation
}

Da Ros, Francesco; Gaiarin, Simone; Zibar, Darko

Published in:

IEEE Photonics Technology Letters

Link to article, DOI:

10.1109/LPT.2019.2946451

Publication date:

2019

Document Version

Peer reviewed version

Link back to DTU Orbit

Citation (APA):

Da Ros, F., Gaiarin, S., \& Zibar, D. (2019). Impact of transmitter phase noise on NFDM transmission with discrete spectral modulation. IEEE Photonics Technology Letters, 31(22), 1767-1770.

https://doi.org/10.1109/LPT.2019.2946451

\section{General rights}

Copyright and moral rights for the publications made accessible in the public portal are retained by the authors and/or other copyright owners and it is a condition of accessing publications that users recognise and abide by the legal requirements associated with these rights.

- Users may download and print one copy of any publication from the public portal for the purpose of private study or research.

- You may not further distribute the material or use it for any profit-making activity or commercial gain

- You may freely distribute the URL identifying the publication in the public portal

If you believe that this document breaches copyright please contact us providing details, and we will remove access to the work immediately and investigate your claim. 


\title{
Impact of transmitter phase noise on NFDM transmission with discrete spectral modulation
}

\author{
Francesco Da Ros, Member, IEEE, OSA, Simone Gaiarin, Member, IEEE, OSA, and Darko Zibar, Member, IEEE
}

\begin{abstract}
Nonlinear frequency division multiplexing (NFDM) systems have been showing remarkable progress in the past few years. However, the majority of the demonstrations have neglected the impact of laser phase noise by employing narrowlinewidth lasers and self-homodyne receivers. The impact of transmitter laser linewidth on NFDM transmission is here numerically and experimentally investigated for dual-polarization discrete NFDM systems. The scaling of linewidth tolerance is analyzed for different signal symbol rates and the results are validated experimentally at $250 \mathrm{MBd}$. Numerical and experimental analysis show a limited degradation due to laser phase noise with relevant penalty appearing only for linewidth-symbol duration products $\left(\Delta \nu \times T_{s}\right)$ above $10^{-3}$. The 250 -MBd experimental results indeed show limited penalty for laser linewidths up to 750 $\mathrm{kHz}$ and $100-\mathrm{kHz}$, for back-to-back and 2000-km transmission, respectively.
\end{abstract}

Index Terms - nonlinear frequency division multiplexing, laser phase noise

\section{INTRODUCTION}

$\mathbf{T}$ HE impressive progress of optical communication systems over the past few decades has brought the systems performance close to the Shannon channel capacity for the linear transmission regime. However, optical fibers are inherently nonlinear and the nonlinear distortion induced by Kerr nonlinearity prevents further enhancing the performance. Several mitigation and compensation techniques have been proposed over the years, both in the digital domain [1], the optical domain [2], and jointly [3], however, no clear winner stands out. A different approach based on a well-established mathematical theory, i.e. the inverse scattering transform (IST), has being gaining momentum as a radically-different promising solution [4]. This approach, more commonly known in the communication community as nonlinear Fourier transform (NFT), focuses on designing a signaling system tailored for the nonlinear fiber channel, i.e. constructively including the impact of Kerr nonlinearity [5]. A number of impressive experimental demonstrations have been reported encoding the information on either the discrete spectrum (solitonic component) [8], the continuous spectrum (dispersive component) [9], or even both simultaneously [10]. Additionally, extensions of nonlinear frequency division multiplexing (NFDM) systems to dual-polarization has also been reported for continuous [11], discrete [12], [13] and joint (continuous and discrete) [14] modulation. aRegardless of these promising demonstration, which showed the potential to apply this signal scheme to practical systems beyond the noiseless lossless scenario

S. Gaiarin, F. Da Ros, and D. Zibar are with the Department of Photonics Engineering, Technical University of Denmark, Kongens Lyngby, 2800 Denmark, e-mail: \{fdro,simga,,dazi $\} @$ fotonik.dtu.dk considered by the IST, a comprehensive investigation of the impact of component imperfections and noise sources both at the transmitter- and receiver-side is still missing. Numerical investigation have mainly focused on neglecting the impact of laser phase noise, whereas experimental demonstration have mainly relied on narrow-linewidth lasers $(\lesssim 1 \mathrm{kHz})$, often in self-homodyne configuration at the receiver side [6], [7], [12], [13]. Only very few preliminary analysis of the performance degradation introduced by laser phase noise have been reported. In particular, in [9] a performance degradation due to local oscillator (LO)'s phase noise and equalizationenhanced phase noise (EEPN) is shown for modulation of the continuous spectrum. The impact of transmitter phase noise on the discrete spectrum is then studied by characterizing numerically the variance of the received spectral amplitudes for an ideal lossless and noiseless system [8]. A similar analysis is performed experimentally in [15] but focused only on on-off eigenvalues modulation. Therefore, it is still unclear how detrimental transmitter laser phase noise is for discrete spectral modulation. In this work, we address this question by reporting a systematic investigation of transmitter laser phase noise for two-eigenvalue dual-polarization NFDM transmission system with quadrature phase-shift keying (QPSK) modulation operating at different transmission rates. The system under investigation is similar to [6]-[8], [11] $-[13]$ and has been chosen as a stronger penalty from phase noise may be expected due to its generally low transmission rate. This letter expands on our initial analysis of [16] through numerical simulations, which extend the investigation to different symbol rates, and a detailed discussion. The remaining of this letter is structured as follows. In Section III, the impact of transmitter laser linewidth on the bit error rate (BER) is numerically investigated in a back-to-back configuration for an ideal transmission system with only laser phase noise and amplified spontaneous emission (ASE) noise as sources of degradation. Then, in Section III] the experimental setup used for the validation of the numerical results is presented. In Section IV, the experimental results are presented and discussed for back-to-back configuration (comparable with the numerical analysis) and for up to 2000-km fiber transmission. Finally, in Section V] the main conclusions are summarized.

\section{NUMERICAL ANALYSIS}

As a first step to evaluate the impact of transmitter phase noise for NFDM systems, a numerical analysis is carried out for an ideal back-to-back configuration. The setup implemented is shown in Fig. 1. At the transmitter side, pseudorandom bit sequences (PRBSs) are generated and mapped 


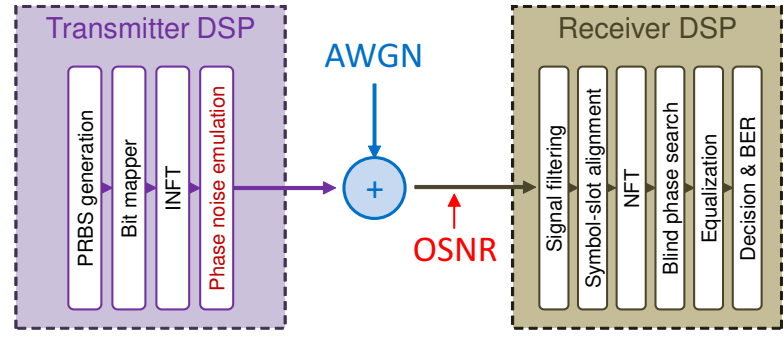

Fig. 1. Setup for the back-to-back numerical simulations.

into QPSK symbols which are directly encoded into the b-coefficients $\left(b\left(\lambda_{i}\right)\right)$ of a two-eigenvalue dual-polarization NFDM signal using only discrete spectral modulation. The choice of eigenvalues $\left(\lambda_{1,2}=\{0.3 i, 0.6 i\}\right)$, and the radii and relative rotation of the symbols constellations follows the optimization discussed in [12], [13] and aimed at limiting the peak-to-average power of the time-domain waveforms, whereas ensuring vanishing boundary conditions [5]. After performing the inverse nonlinear Fourier transform (INFT) operation, the time domain waveforms (256 Samples/symbol) are ideally encoded onto an optical carrier, i.e. assuming an ideal digital-to-analog converter (DAC) and modulator. The only physical effect taken into account is the laser phase noise which is emulated (phase noise emulation block in Fig. 1) as a first-order Wiener process [17] with the desired linewidth:

$$
\Phi_{k}=\Phi_{k-1}+\sigma \cdot n_{k},
$$

where $\Phi_{k}$ is the phase noise at instant $k$, and $n_{k}$ 's are independent identically distributed Gaussian variables with zero mean and unitary variance. The pre-factor $\sigma^{2}$ scales the Gaussian noise variables to the desired variance defined as $\sigma^{2}=2 \cdot \pi \cdot \Delta \nu / F_{s}$, with $\Delta \nu$ the transmitter laser linewidth and $F_{s}$ the sampling frequency.

After phase noise loading, complex additive white Gaussian noise (AWGN) is added to the optical signal according to the desired optical signal-to-noise ratio (OSNR) defined over the customary $12.5 \mathrm{GHz}$ bandwidth. At the receiver side, an ideal coherent receiver is assumed to give direct access to the complex electrical field. No LO phase noise is considered in this work, in order to focus on the impact of the transmitter's alone. Preliminary results on the relevance of LO phase noise can be found in [9] for continuous spectral modulation. Digital signal processing consisting of low-pass filtering and crosscorrelation based time-recovery for symbol slot alignment is applied before extracting the discrete spectral components through a direct NFT operation, obtaining directly the symbols $\left(b\left(\lambda_{i}\right)\right)$ at 1 Sample/symbol. According to the property of the NFT operation [5], a constant phase rotation in time is directly translated into a negative phase rotation of the bcoefficient $e^{i \Phi} s(t) \Longleftrightarrow e^{-i \Phi} b(\lambda)$. Therefore, as long as the phase noise process varies slowly within a single symbol slot $\left(\Delta \nu \times T_{s} \ll 1\right)$, phase recovery can be performed directly in the spectral domain. In our analysis we choose blind phase search (BPS) [18] as a simple and nevertheless effective scheme. The number of test phases for BPS has been kept constant to 16 throughout the analysis, whereas the averaging factor has been optimized considering the trade-off between signal OSNR (need for averaging) and transmitter laser linewidth (tracking speed). After carrier recovery, signal equalization based on a linear minimum mean square error (LMMSE) estimation is performed [13] followed by BER counting. Notice BPS and LMMSE operate at $1 \mathrm{Sa} /$ symbol, i.e. tracking every $T_{s}$. The choice of a fairly ideal setup without proper modeling of electro-optic and opto-electronic conversion is dictated by our desire to focus only on the impact of laser phase noise in combination with ASE noise. Practical component limitations are then taken into account in the experimental investigation of Sections III and IV]

The BER performance as a function of the received OSNR is shown in Fig. 2 for different transmitter laser linewidths and signal baudrates. Remark that the signal have relatively high OSNR requirements even for the low baudrates considered as the time-bandwidth product has not been optimized [19], therefore a $250-\mathrm{MBd}$ signals has a $20-\mathrm{dB}$ bandwidth of approx. $3.3 \mathrm{GHz}$, and the signal bandwidth scales linearly with the baudrate. The number of samples per symbol has been kept constant (256 Samples/symbol) for the different baudrates to keep fixed any additional penalty which may arise from a lower precision of INFT and NFT operations. Sequences of $2^{18}$ bits have been simulated, leading to reliable BER values above $4 \times 10^{-5}$.

The results show a high tolerance to transmitter laser linewidth which increases with the signal baudrate, as expected. The OSNR penalty is within $1 \mathrm{~dB}$ at a BER of $1 \times 10^{-4}$ for up to $250 \mathrm{kHz}$ of linewidth at $250 \mathrm{MBd}\left(\Delta \nu \times T_{s}=0.001\right)$ and such number scales linearly with the baudrate: approx. $500 \mathrm{kHz}$ at $500 \mathrm{MBd}$ and beyond $750 \mathrm{kHz}$ at $1 \mathrm{GBd}$, i.e. confirming a rough threshold of $\Delta \nu \times T_{s} \approx 0.001$.

\section{EXPERIMENTAL SETUP}

The numerical setup of Fig. 1 is extended for the experimental validation to Fig. 3 . After the transmitter-side digital signal processing (DSP), i.e. right after the phase noise emulation block, the waveforms are loaded into a 64-GSa/s arbitrary waveform generator (AWG) driving a dual-polarization IQ modulator. The digital waveforms are encoded onto an optical carrier originating from a narrow-linewidth laser $(50 \mathrm{~Hz}$, correlation length $\approx 2000 \mathrm{~km}$ ). As only emulated linewidths above $2 \mathrm{kHz}$ are considered, the main contribution to the transmitter phase noise comes from the digitally emulated laser phase noise. Whereas for the transmission analysis, the signal is then injected into a recirculating transmission loop, for the initial back-to-back characterization the signal is directly fed to the receiver with a noise loading stage (red stage in Fig. 3). ASE noise from an erbium-doped fiber amplifier (EDFA) is used to vary the received signal OSNR. After noise loading, the signal is received by a standard pre-amplified coherent receiver in homodyne configuration, i.e. using the transmitter laser as LO to avoid phase noise and random frequency offset contributions from the receiver side and ensure control of the emulated laser linewidth. This choice allows focusing on the impact of transmitter phase noise, by removing the degradation due to EEPN. After analog-to-digital conversion in a $80-\mathrm{GSa} / \mathrm{s}$ 

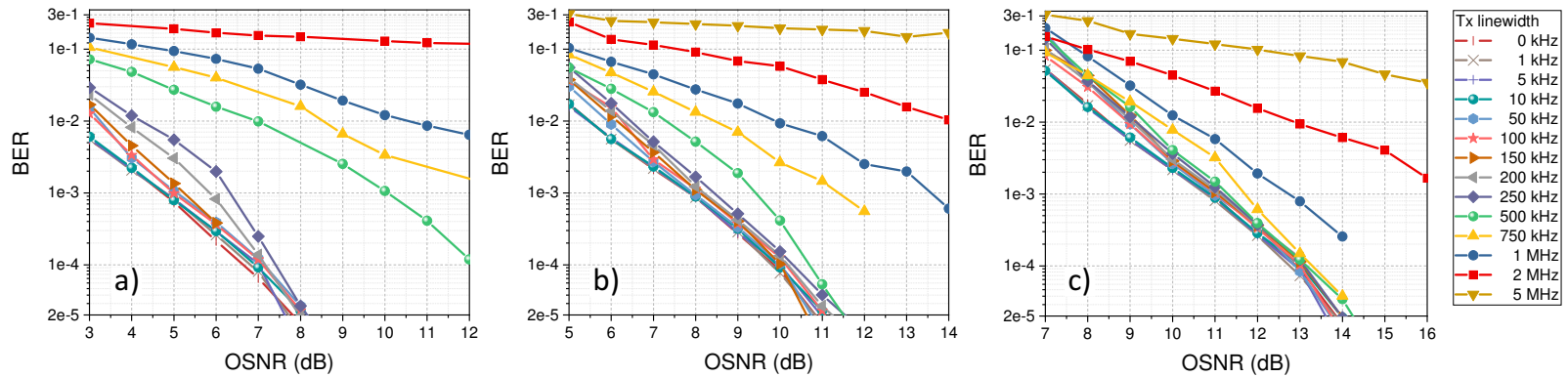

Fig. 2. Back-to-back BER as a function of received OSNR for different transmitter laser linewidths: (a) 250-MBd, (b) 500-MBd, and (c) 1-GBd signals.

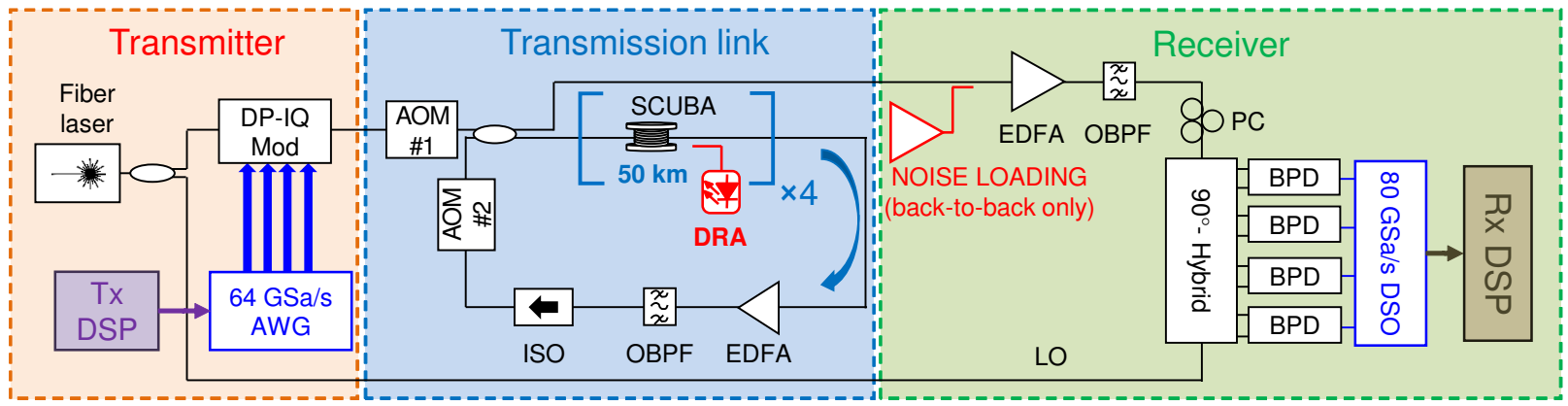

Fig. 3. Experimental setup for back-to-back and fiber transmission investigations.

digital storage oscilloscope (DSO), the receiver-side digital signal processing (DSP) described in Section $\Pi$ is performed. Alternatively, for the transmission analysis, the recirculating transmission loop consists of 4 spools of 50-km long lowloss large-effective area submarine fiber (OFS SCUBA fiber, $D=22 \mathrm{ps} / \mathrm{nm} / \mathrm{km}, A_{\text {eff }}=153 \mu^{2}, \alpha=0.155 \mathrm{~dB} / \mathrm{km}$ ) using distributed Raman amplification (DRA) with backward pump to minimize the power variations throughout the transmission [13], [14]. The loop operation is controlled through acustooptic modulators (AOM) acting as shutters and an EDFA followed by a optical band pass filter (OBPF) compensates for the additional loop loss. As the AOMs introduce a static frequency up-shift, for the transmission experiment, a constant frequency down-shift is performed as first processing step in the receiver-side DSP.

\section{EXPERIMENTAL RESULTS}

The experimental analysis focuses on a 250-MBd signal and the results for back-to-back and transmission configurations are reported in Subsection IV-A and IV-B, respectively. Due to memory restriction in the AWG symbols sequences of only 1024 symbols could be transmitted. The results reported have then being averaged over different phase and ASE noise realizations for statistical significance.

\section{A. Back-to-back}

Fig. 4 shows the BER performance in back-to-back configuration as a function of the received OSNR tuned by ASE noise loading. The performance are reported for several emulated laser linewidths and as expected, the BER indeed degrades with the laser linewidth, ultimately resulting in error floors at high BER levels. However, the appearance of such

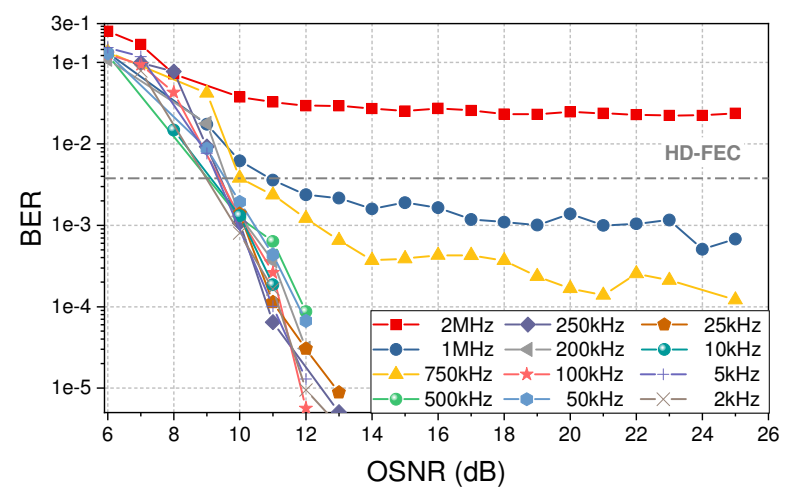

Fig. 4. Back-to-back performance of a 250-MBd NFDM signal as a function of the received OSNR for different transmitter laser linewidth.

floors takes place only at rather large linewidth values above $750 \mathrm{kHz}$, i.e. a $\Delta \nu \times T_{s}=0.003$. This compares well with the numerical results of Fig. 2, actually showing that realistic implementations show slightly lower penalty due to phase noise compared to the simulations. This is the results of more dominant degradation due to the noise and distortion sources within transmitter and receiver which were ignored in the numerical analysis of Section III Indeed, the experimental curves are shifted to higher OSNR values compared to the numerical simulations of the ideal setup of Fig. 1 1 even at very low transmitter laser linewidths. These results show that the impact of laser phase noise is far from being a dominant cause of degradation for NFDM systems.

\section{B. Transmission results}

Fig. 5 shows the BER performance as a function of the laser linewidth after $1000 \mathrm{~km}, 1600 \mathrm{~km}$ and $2000 \mathrm{~km}$ transmission. 


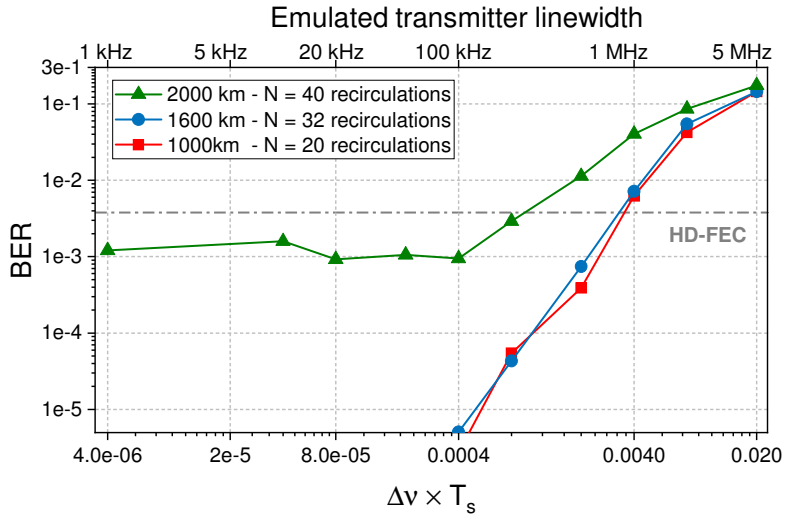

Fig. 5. BER performance of a $250-$ MBd NFDM signal as a function of the transmitter laser linewidth for 1000,1600 and $2000 \mathrm{~km}$ transmission.

Comparing $1000 \mathrm{~km}$ and $1600 \mathrm{~km}$, almost identical performance can be seen, at least for the region of relevant BER values, i.e. where transmitter phase noise is the main limitation. Only at longer distances $(2000 \mathrm{~km})$, the performance degrade due to loss and ASE, similarly to the results of [13]. Remark that only distances below the coherence length of our laser $(\approx 2000 \mathrm{~km})$ are considered, thus making the emulated phase noise the dominant contribution.

As the linewidth is increased, the degradation due to phase noise is more detrimental compared to the back-to-back case. Unlike for conventional (non-NFT-based) systems, in NFDM, noise and power variations contribute to distortion of the b-coefficients in two ways: complex noise is added to the symbols, and additional phase noise is generated due to eigenvalue displacement. The displacement leads to a rotation of the constellations [13], [20], further increasing the phase variance of the b-coefficients. Nevertheless, the 250-MBd signal shows very limited degradation for up to $100 \mathrm{kHz}$ of linewidth. Whereas such a value can be achieved with current laser technology, the impact of phase noise decreases with the baudrate also for NFDM systems as shown in Fig. 2. Therefore, moving to higher baud rates is expected to further relax the requirements on the transmitter laser linewidth. Finally, as mentioned, only a simple phase recovery scheme (BPS) was used in this work. More refined phase noise tracking schemes than BPS, could further reduce the degradation. Phase recovery tailored for NFDM systems and performed directly on the time-domain waveforms before the NFT operation may allow to relax the assumption of slowly varying phase noise over the symbol slot, thus enabling a more accurate estimation.

\section{CONCLUSION}

We investigated the impact of transmitter phase noise on the BER performance on dual-polarization NFDM systems using discrete spectral modulation. A numerical analysis at different baudrates showed a large tolerance to phase noise with OSNR penalties above $1 \mathrm{~dB}\left(\mathrm{BER}=1 \times 10^{-4}\right)$ appearing only for $\Delta \nu \times T_{s}>0.001$. Further experimental validation for low baudrate signals $(250 \mathrm{MBd})$ confirmed the trends from the numerical investigation and showed that the penalty from the transmitter laser linewdith is limited for linewidths up to 750
$\mathrm{kHz}$ in back-to-back configuration and for up to $100 \mathrm{kHz}$ for up to $2000-\mathrm{km}$ transmission. The higher tolerance reported experimentally shows that transmitter laser phase noise is currently far from being a dominant source of penalty for NFDM systems. Further investigation is however needed to evaluate the impact of EEPN.

\section{ACKNOWLEDGMENT}

This work is supported by the European Research Council (ERC-CoG FRECOM project, 771878). We thank OFS fitel Denmark for providing the SCUBA fiber used in this work.

\section{REFERENCES}

[1] E. Ip, and J. Kahn, "Compensation of Dispersion and Nonlinear Impairments Using Digital Backpropagation,” J. Light. Technol., 26(20), 34163425 (2008).

[2] I. Sackey, et al., "Kerr nonlinearity mitigation in 5×28-GBd PDM 16QAM signal transmission over a dispersion-uncompensated link with backward-pumped distributed Raman amplification," Opt. Expr., 22, 27381-27391 (2014).

[3] M.P. Yankov, et al., "Experimental comparison of probabilistic shaping with online PMF optimization and mid-link OPC," in Proc. CLEO 2018, paper STu4C.2.

[4] V.E. Zakharov and A.B. Shabat, "Exact theory of 2-dimensional selffocusing and one-dimensional self-modulation of waves in nonlinear media," Sov. Phys. JETP 34, 62-69 (1972).

[5] M.I. Yousefi and F.R. Kschischang, "Information transmission using the nonlinear Fourier transform, part I: mathematical tools," IEEE Trans. Inf. Theory, 60, 4312-4328 (2014)

[6] A. Gejsler, et al., "Influence of non-ideal first order counter-propagating raman amplification on discrete nonlinear Fourier spectrum based communication," in Proc. 19th ITG-Symposium (2018).

[7] V. Aref, et al., "Experimental demonstration of nonlinear frequency division multiplexed transmission," in Proc. ECOC 2015, paper 0372.

[8] T. Gui, et al., "Phase Modulation on Nonlinear Discrete Spectrum for Nonlinear Frequency Division Multiplexed Transmissions," in Proc. OFC 2016, W3A.2.

[9] S.T. Le, et al., "Equalization-Enhanced Phase Noise in Nonlinear Inverse Synthesis Transmissions," in Proc. ECOC 2016, pp. 370.

[10] S.T. Le, et al., "Nonlinear signal multiplexing for communication beyond the Kerr nonlinearity limit" Nat. Phot., 11, 570-576 (2017).

[11] W.A. Gemechu, et al., "Dual polarization nonlinear frequency division multiplexing transmission," IEEE Phot. Tech. Lett., 30 1589-1592 (2018).

[12] S. Gaiarin, et al., "Dual-polarization nonlinear Fourier transform-based optical communication system" Optica 5(3), 263-270 (2017).

[13] S. Gaiarin, et al., "Dual-polarization NFDM transmission using distributed Raman amplification and NFT-domain equalization," IEEE Phot. Tech. Lett., 30, 1983 (2018)

[14] F. Da Ros, et al., 'Dual-Polarization NFDM Transmission With Continuous and Discrete Spectral Modulation," J. Light. Technol. 37(10), 23352343 (2019).

[15] Z. Dong, et al., "Impact of Frequency Offset and Laser Phase Noise on Nonlinear Frequency Division Multiplexed Systems via the Nonlinear Fourier Transform," in Proc. ACP 2015, ASu3F.2.

[16] F. Da Ros, et al., "Impact of laser phase noise on nonlinear frequency division multiplexing systems," in Proc. CLEO 2019, paper SW3O.6.

[17] A. Demir, et al., "Phase noise in oscillators: a unifying theory and numerical methods for characterization," IEEE Trans. Circuits Syst. I, 47, 655-674 (2000).

[18] T. Pfau, et al., "Hardware-efficient coherent digital receiver concept with feedforward carrier recovery for M-QAM constellations," J. Light. Technol. 27(8), 989-999 (2009).

[19] A. Span, et al., "Time-Bandwidth Product Perspective for Nonlinear Fourier Transform based Multi-Eigenvalue Soliton Transmission," IEEE Trans. on Comm., early access DO:10.1109/TCOMM.2019.2913870 (2019).

[20] T. Gui, et al., "Alternative decoding methods for optical communications based on nonlinear Fourier transform," J. Light. Technol., 35(9), 1542 1550 (2017). 